\title{
Mixture Random Hypersurface Models for Tracking Multiple Extended Objects
}

\author{
Marcus Baum, Benjamin Noack, and Uwe D. Hanebeck
}

\begin{abstract}
This paper presents a novel method for tracking multiple extended objects. The shape of a single extended object is modeled with a recently developed approach called Random Hypersurface Model (RHM) that assumes a varying number of measurement sources to lie on scaled versions of the shape boundaries. This approach is extended by introducing a socalled Mixture Random Hypersurface Model (Mixture RHM), which allows for modeling multiple extended targets. Based on this model, a Gaussian-assumed Bayesian tracking method that provides the means to track and estimate shapes of multiple extended targets is derived. Simulations demonstrate the performance of the new approach.
\end{abstract}

Keywords: Multiple Extended Object Tracking, Shape Tracking, Random Hypersurface Model

\section{INTRODUCTION}

In contrast to a point target, an extended object may be the origin of several measurements from different measurement sources on its surface (see Fig. 1). Essentially, there are two major scenarios that illustrate this issue. First, continuously evolving sensor technologies provide advanced resolution capabilities that can result into several measurements of one target during a single scan. The measurement sources vary from scan to scan and their locations depend on the shape of the target but also on more complex target-dependent properties. Second, a group of point targets can also be treated as a single entity when there are strong interdependencies between the individual group members.

In this work, we consider the problem of tracking multiple extended objects (see Fig. 1), where the goal is to estimate a shape approximation of each extended target in addition to its kinematic parameters [1]-[3]. Note that this is a nontrivial task, as it is required to deal with measurements that may stem from different objects whose extents are unknown and are part of the estimation problem.

In order to model the shape of a single extended target, we employ a recent approach called Random Hypersurface Model (RHM) [3], [4]. An RHM assumes that measurement sources lie on scaled versions of the shape boundary, which allows for estimating the form and the extent of the shape. In doing so, the target can be modeled as a basic shape, such as an ellipse [3], or even as an arbitrary star-convex shape [4]. So far, RHMs are restricted to a single extended target.

The main contribution of this paper is a method for modeling and tracking multiple extended targets based on

Marcus Baum, Benjamin Noack, and Uwe D. Hanebeck are with the Intelligent Sensor-Actuator-Systems Laboratory (ISAS), Institute for Anthropomatics, Karlsruhe Institute of Technology (KIT), Germany. marcus.baum@kit.edu, noack@kit.edu, uwe.hanebeck@ieee.org

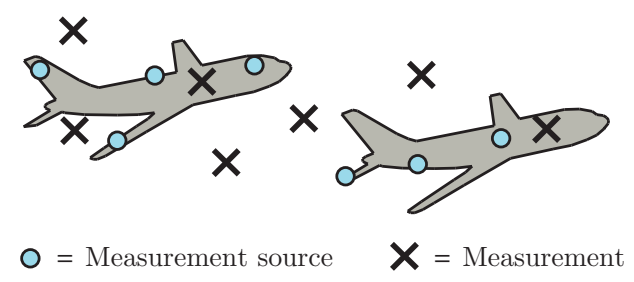

Fig. 1: Two closely-spaced extended objects.

$R H M s$. For this purpose, we first introduce the novel concept of Mixture Random Hypersurface Models (Mixture RHMs), which are an extension of RHMs to multiple extended targets. Based on this model, we derive a Bayesian method for tracking multiple extended targets and we suggest a particular implementation as a Gaussian-assumed density filter.

The remainder of this paper is structured as follows: First, we give a brief overview of related approaches for extended object tracking in Section II. Afterwards, in Section III we discuss how a single extended object can be modeled with RHMs. Subsequently, we introduce the basic idea of Mixture Random Hypersurface Models in Section IV-A and derive a Bayesian estimator for Mixture RHMs in Section V. Simulations in Section VI demonstrate the feasibility of the approach. Finally, this paper is concluded in Section VII.

\section{RELATED WORK}

Spatial distribution models [1], [5] assume that each measurement source is randomly drawn from a known objectdependent probability distribution. In [1], [5], [6], spatial distribution models have been utilized to track multiple extended targets, e.g., stick targets, in a cluttered environment. Recently, spatial distribution models have been integrated into Probability Hypothesis Density (PHD) filters for tracking multiple extended objects [7], [8]. In [9], a Sequential Monte-Carlo (SMC) approach for tracking extended objects based on border parameterization is presented. A recent approach for modeling elliptic target extents is based on random symmetric positive definite matrices [10], [11], which has also been integrated into the Probabilistic Multiple-Hypothesis Tracker (PMHT) framework [12] for tracking multiple extended targets. A thorough comparison of the random matrix approach with Random Hypersurface Models can be found in [13]. 


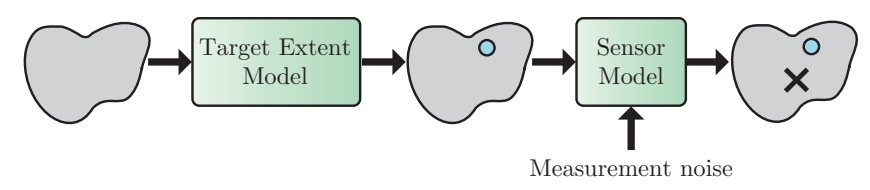

Fig. 2: Measurement model for extended objects.

\section{Modeling Single Extended TARgets with RHMS}

In order to track a shape approximation of a single extended object [4], we need to define the state to be estimated, a measurement model (see Section III-A), and a system model (see Section III-B).

An extended target is defined as a set $\mathcal{S}\left(\underline{p}_{k}\right)$, where $\underline{p}_{k}$ is the parameter vector for $\mathcal{S}\left(\underline{p}_{k}\right)$. For example in case of a circular target, $\underline{p}_{k}$ consists of the radius and center of the extended object and $\mathcal{S}\left(\underline{p}_{k}\right)$ are all the points forming the circular disc. The stacked state vector of an extended target at time step $k$ to be tracked is a random vector $\underline{\boldsymbol{x}}_{k}=\left[\underline{\boldsymbol{p}}_{k}^{T}, \underline{\boldsymbol{r}}_{k}^{T}\right]^{T}$ that consists of the parameter vector $\underline{\boldsymbol{p}}_{k}$ and possible further state variables subsumed under the vector $\underline{\boldsymbol{r}}_{k}$, e.g., the velocity.

\section{A. Measurement Model}

At each time step $k$, a set of $n_{k}$ position measurements $\left\{\underline{\hat{y}}_{k, l}\right\}_{l=1}^{n_{k}}$ is available. We assume that the measurements are generated independently and that they all stem from the target. For given $\mathcal{S}\left(\underline{p}_{k}\right)$, the measurement model specifies how a single measurement $\underline{\hat{y}}_{k, l}$ is obtained. The measurement model is composed of two successive parts, the target extent model and the sensor model (see Fig. 2) as discussed in the following.

Target Extent Model: For a given shape $\mathcal{S}\left(\underline{p}_{k}\right)$, the target extent model determines where the measurement source $\underline{z}_{k, l}$ is located on the object (see Fig. 2). We employ an RHM for the target extent, which is described in Section III-C.

Sensor Model: Given a measurement source $\underline{z}_{k, l}$, the sensor model specifies how the measurement $\underline{\hat{y}}_{k, l}$ is obtained. Here, we restrict ourselves to position measurements, i.e.,

$$
\underline{\hat{y}}_{k, l}=\underline{z}_{k, l}+\underline{\boldsymbol{v}}_{k, l},
$$

where the noise term $\underline{\boldsymbol{v}}_{k, l}$ is a zero-mean white Gaussian noise with covariance matrix $\Sigma_{k, l}^{v}$.

\section{B. Dynamic Model}

The temporal evolution of the target state $\underline{\boldsymbol{x}}_{k}$ is characterized by a system equation of the form

$$
\underline{\boldsymbol{x}}_{k+1}=a_{k}\left(\underline{\boldsymbol{x}}_{k}, \underline{u}_{k}, \underline{\boldsymbol{w}}_{k}\right),
$$

where $a_{k}(\cdot)$ is the system function, $\underline{u}_{k}$ is the system input, and $\underline{\boldsymbol{w}}_{k}$ the system noise. As the shape vector is part of the state vector, this model also incorporates the temporal evolution of the shape.

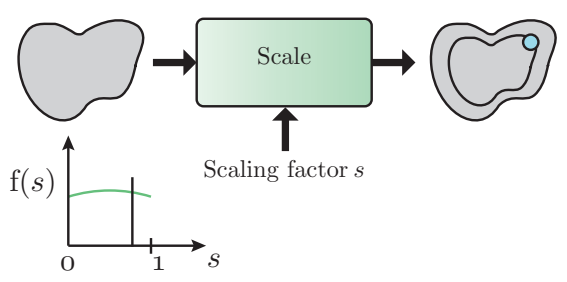

Fig. 3: Random Hypersurface Model.

\section{Random Hypersurface Model (RHM)}

A Random Hypersurface Model [3], [4], [14] is a target extent model, i.e., it characterizes the location of a single measurement source for a given shape. In the following, we use a particular RHM tailored to star-convex shapes as described in [4]. Intuitively, a set is star-convex if it contains a specific point from which each other point in the set is in the line-of-sight [4].

For a given star-convex shape, an $R H M$ assumes the measurement source is an element of a randomly scaled version of the shape boundary (see Fig. 3). The scaling factor is randomly drawn from a one-dimensional probability density function, which has to be specified in advance.

Definition III.1 (Random Hypersurface Model (RHM)) Let $\overline{\mathcal{S}}\left(\underline{p}_{k}\right)$ be the boundary of the star-convex set $\mathcal{S}\left(\underline{p}_{k}\right)$ with center $\underline{m}_{k}$, then the measurement source $\underline{z}_{k, l}$ is generated according to an $R H M$, if $\underline{z}_{k, l}$ is an element of the scaled boundary, i.e.,

$$
\underline{z}_{k, l} \in \underline{m}_{k}+\tilde{s}_{k, l} \cdot\left(\overline{\mathcal{S}}\left(\underline{p}_{k}\right)-\underline{m}_{k}\right),
$$

where $\tilde{s}_{k, l}$ is a random draw from the one-dimensional random variable $s_{k, l} \in[0,1]$ and the algebraic operations ",,.+- " are meant element-wise.

Note that the restriction to star-convex shapes makes sure that $\underline{z}_{k, l} \in \mathcal{S}\left(\underline{p}_{k}\right)$.

\section{Star-Convex RHM Using a Radius Function}

In the following, we briefly summarize the representation of a star-convex shape by means of a radius function suggested in [4]. Based on this representation and the concept of an RHM, a measurement equation can be derived.

1) Representation of a Star-Convex Shape: A star-shaped extended object $\mathcal{S}\left(\underline{p}_{k}\right)$ can be represented in parametric form with a so-called radius function $r(\phi)$ [15], [16], which encodes the distance from the center to a contour point depending on the angle $\phi$.

We suggest to parameterize the radius function by means of the Fourier coefficients. If it is considered as periodic function in $\phi$ with period $[0,2 \pi]$, the (truncated) Fourier series expansion becomes

$$
r\left(\underline{b}_{k}, \phi\right)=\frac{a_{k}^{(0)}}{2}+\sum_{j=1 \ldots N_{F}} a_{k}^{(j)} \cos (j \phi)+b_{k}^{(j)} \sin (j \phi),
$$

where the parameter vector

$$
\underline{b}_{k}=\left[a_{k}^{(0)}, a_{k}^{(1)}, b_{k}^{(1)}, \ldots a_{k}^{\left(N_{F}\right)}, b_{k}^{\left(N_{F}\right)}\right]^{T}
$$


contains the Fourier coefficients. Note that (3) is linear in $\underline{b}_{k}$ for fixed $\phi$, i.e., $r\left(\underline{b}_{k}, \phi\right)=\mathbf{R}(\phi) \cdot \underline{b}_{k}$, where

$$
\mathbf{R}(\phi)=\left[1, \cos (\phi), \sin (\phi), \ldots, \cos \left(N_{F} \phi\right), \sin \left(N_{F} \phi\right)\right] .
$$

The complete shape parameter vector is $\underline{p}_{k}=\left[\underline{b}_{k}^{T}, \underline{m}_{k}^{T}\right]^{T}$, where $\underline{m}_{k}$ is the center of object. The extended object can be written as

$$
\begin{aligned}
\mathcal{S}\left(\underline{p}_{k}\right)=\left\{s \cdot r\left(\underline{b}_{k}, \phi_{k}\right) \cdot \underline{e}\left(\phi_{k, l}\right)+\underline{m}_{k} \mid\right. \\
\left.\phi_{k} \in[0,2 \pi] \text { and } s \in[0,1]\right\}
\end{aligned}
$$

in which $\underline{e}\left(\phi_{k, l}\right):=\left[\cos \left(\phi_{k, l}\right), \sin \left(\phi_{k, l}\right)\right]^{T}$ is the unit vector with angle $\phi_{k, l}$.

2) Measurement Equation: Based on the particular shape representation and the concept of an RHM, it is possible to state the measurement equation

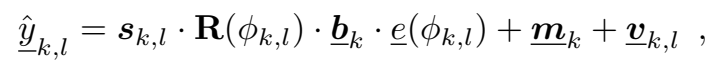

which maps the state vector $\underline{\boldsymbol{x}}_{k}$, the measurement noise $\underline{\boldsymbol{v}}_{k, l}$, and the scaling factor $s_{k, l}$ to the measurement $\underline{\hat{y}}_{k, l}$. In (5), the term $\phi_{k, l}$ denotes the unknown angle between the vector from the center to the measurement source $\underline{z}_{k, l}$ and the $x$ axis. As $\phi_{k, l}$ is unknown, we suggest to replace it with a point estimate, e.g., the most likely angle $\phi_{k, l}$. In case of isotropic measurement noise, a proper point estimate $\phi_{k, l}$ is given by the angle between the vector from the current shape center estimate $\underline{\mu}_{k, l-1}^{m}$ to the measurement $\underline{\hat{y}}_{k, l}$ and the $x$-axis.

An algebraic reformulation of (5) as described in [4] can used for reducing the effect of $\phi_{k, l}$ and yield the final measurement equation

$$
\begin{aligned}
0= & h^{*}\left(\underline{\boldsymbol{x}}_{k}, \underline{\boldsymbol{v}}_{k, l}, \boldsymbol{s}_{k, l}, \underline{\hat{y}}_{k, l}\right) \\
:= & \boldsymbol{s}_{k, l}^{2} \cdot\left\|\mathbf{R}\left(\hat{\phi}_{k, l}\right) \cdot \underline{\boldsymbol{b}}_{k}\right\|^{2}+ \\
& 2 \boldsymbol{s}_{k, l} \mathbf{R}\left(\hat{\phi}_{k, l}\right) \underline{\boldsymbol{b}}_{k} \underline{e}\left(\hat{\phi}_{k, l}\right)^{T} \underline{\boldsymbol{v}}_{k, l}+\left\|\underline{\boldsymbol{v}}_{k, l}\right\|^{2}- \\
& \left\|\underline{\hat{y}}_{k, l}-\underline{\boldsymbol{m}}_{k}\right\|^{2},
\end{aligned}
$$

where $h^{*}\left(\underline{\boldsymbol{x}}_{k}, \underline{\boldsymbol{v}}_{k, l}, \boldsymbol{s}_{k, l}, \underline{\hat{y}}_{k, l}\right)$ maps the state vector $\underline{\boldsymbol{x}}_{k}$, scaling factor $\boldsymbol{s}_{k, l}$, measurement noise $\underline{\boldsymbol{v}}_{k, l}$, and measurement $\underline{\hat{y}}_{k, l}$ to a so-called pseudo-measurement 0 .

\section{Modeling Multiple Extended Targets with MiXTURE RHMS}

In this section, we introduce a novel concept called Mixture Random Hypersurface Model (Mixture RHM), which can be used for modeling multiple extended objects. In the following, we assume that there are $N$ extended targets

$$
\mathcal{S}\left(\underline{p}_{k}^{1}\right), \ldots, \mathcal{S}\left(\underline{p}_{k}^{N}\right)
$$

and at each time step $k$, a set of $n_{k}$ measurements $\left\{\underline{\hat{y}}_{k, l}\right\}_{l=1}^{n_{k}}$ is available. It is not known, which measurement stems from which extended target. In order to estimate the parameters $\underline{p}_{k}^{1}, \ldots, \underline{p}_{k}^{N}$, we need a model that relates the parameter vectors with the measurements. This model is obtained by extending the concept of RHMs to mixtures of RHMs (which is then called Mixture Random Hypersurface Model) as described in the following.

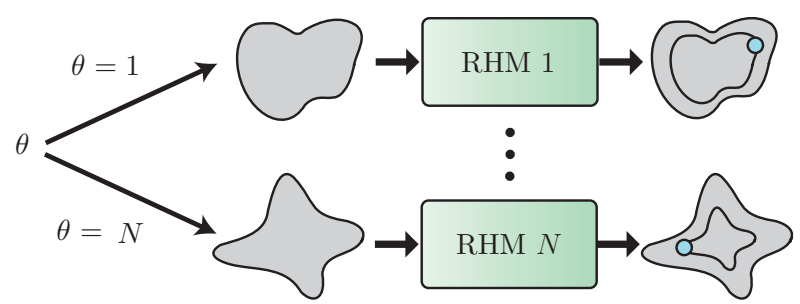

Fig. 4: Mixture Random Hypersurface Model.

\section{A. Mixture Random Hypersurface Model}

A Mixture RHM is defined in analogy to classical mixture models such as Gaussian mixture models. For a given set of $N$ extended objects

$$
\mathcal{S}\left(\underline{p}_{k}^{1}\right), \ldots, \mathcal{S}\left(\underline{p}_{k}^{N}\right),
$$

a Mixture RHM specifies the generation of a single measurement source $\underline{z}_{k, l}$. Intuitively, the measurement source lies on the scaled boundary of an extended object that is selected according to a discrete association random variable $\boldsymbol{\theta}_{k, l} \in\{1, \ldots N\}$. A visualization of a Mixture $R H M$ is depicted in Fig. 4 and a more formal definition is given in the following.

\section{Definition IV.1 (Mixture Random Hypersurface Model)}

A Mixture RHM for $N$ extended targets $\mathcal{S}\left(\underline{p}_{k}^{1}\right), \ldots, \mathcal{S}\left(\underline{p}_{k}^{N}\right)$ consists of

- a discrete random variable $\boldsymbol{\theta}_{k, l} \in\{1, \ldots N\}$, which is called association variable, and

- $N$ Random Hypersurface Models with scaling factors $\boldsymbol{s}_{k, l}^{1}, \ldots, \boldsymbol{s}_{k, l}^{N}$.

A measurement source $\underline{z}_{k, l}$ is drawn according to a Mixture RHM iff

$$
\underline{z}_{k, l} \in \underline{m}_{k}^{\theta}+\tilde{s}_{k, l}^{\theta} \cdot\left(\overline{\mathcal{S}}\left(\underline{p}_{k}^{\theta}\right)-\underline{m}_{k}^{\theta}\right)
$$

where

- $\theta$ is a random draw from $\boldsymbol{\theta}_{k, l}$, which specifies a particular extended object,

- $\underline{p}_{k}^{\theta}$ is the parameter vector of the selected extended object,

- $\underline{m}_{k}^{\theta}$ is the center of the selected extended object,

- $\tilde{s}_{k, l}^{\theta}$ is a random draw from the scaling factor $s_{k, l}^{\theta}$, and

- $\overline{\mathcal{S}}\left(\underline{p}_{k}^{\theta}\right)$ denotes the boundary of $\mathcal{S}\left(\underline{p}_{k}^{\theta}\right)$.

In the following, we assume that a Mixture RHM generates measurements independently, i.e., the association variables and scaling factors are independent. Due to this assumption, the combinatorial complexity of the data association problem is dramatically reduced. There exist several multitarget tracking approaches such as the Probabilistic MultipleHypothesis Tracker (PMHT) [12], [17] or [1], [5] that make similar assumptions.

A Mixture RHM can be seen as the basic concept for modeling multiple extended objects, which can be equipped and combined with additional target-related or sensor specific 
modeling knowledge. For instance, the number of measurements received from a target can be assumed to be drawn from a Poisson distribution. This assumption can be incorporated into Mixture RHMs by selecting suitable association probabilities (see [1], [5]). Also, a background clutter model as described in [1], [5] can be added. Of course, Mixture RHMs can also be combined with models for other target types, e.g., point targets. If required, we could also drop the assumption of independently generated measurements. In this case, one has to evaluate all possible association hypotheses.

\section{BAYESian Tracking of Multiple Extended TARGETS}

Based on the Mixture Random Hypersurface Model, the sensor model (1), and the system model (2) for the extended objects, a recursive Bayesian state estimator for multiple extended objects can be derived. We consider the stacked random vector $\underline{\boldsymbol{x}}_{k}^{A}$ of all $N$ target states

$$
\underline{\boldsymbol{x}}_{k}^{A}=\left[\begin{array}{c}
\underline{\boldsymbol{x}}_{k}^{1} \\
\vdots \\
\underline{\boldsymbol{x}}_{k}^{N}
\end{array}\right] .
$$

The posterior probability density function of $\underline{\boldsymbol{x}}_{k}^{A}$ having received all measurements up to time step $k-1$ and the measurements $\underline{\hat{y}}_{k, 1}, \ldots, \underline{\hat{y}}_{k, l}$ from time step $k$ is denoted by $f_{k, l}\left(\underline{x}_{k}^{A}\right)$.

Time Update: From the system equation (2) for the individual extended targets, we can derive a conditional density function $f\left(\underline{x}_{k}^{A} \mid \underline{x}_{k-1}^{A}\right)$. Hence, the prediction $f_{k, 0}\left(\underline{x}_{k}\right)$ for time step $k$ is given by the Chapman-Kolomogorov equation

$$
f_{k, 0}\left(\underline{x}_{k}^{A}\right)=\int f\left(\underline{x}_{k}^{A} \mid \underline{x}_{k-1}^{A}\right) \cdot f_{k-1, n_{k-1}}\left(\underline{x}_{k-1}^{A}\right) d \underline{x}_{k-1}^{A} .
$$

Measurement Update: The prediction $f_{k, 0}\left(\underline{x}_{k}\right)$ is updated with the received measurements $\left\{\underline{y}_{k, l}\right\}_{l=1}^{n_{k}}$ according to Bayes' rule. Because the measurement generation based on a Mixture RHM is assumed to be independent for consecutive measurements, the update can be conducted recursively. Hence, given the probability density $f_{k, l-1}\left(\underline{x}_{k}^{A}\right)$ and the measurement $\underline{y}_{k, l}$, the goal is to compute the posterior density

$$
f_{k, l}\left(\underline{x}_{k}^{A}\right)=f_{k, l-1}\left(\underline{x}_{k}^{A} \mid \underline{\hat{y}}_{k, l}\right) .
$$

The major challenge in deriving these expressions is the data association uncertainty, i.e., it is unknown from which extended object $\underline{\boldsymbol{x}}_{k}^{i}$ the measurement $\underline{\hat{y}}_{k, l}$ stems.

According to the Law of Total Probability, (7) can be written as

$$
\begin{aligned}
& f_{k, l}\left(\underline{x}_{k}^{A}\right)= \\
& \sum_{\theta_{k, l}=1, \ldots, N} f_{k, l-1}\left(\underline{x}_{k}^{A} \mid \theta_{k, l}, \underline{\hat{y}}_{k, l}\right) \cdot f_{k, l-1}\left(\theta_{k, l} \mid \underline{\hat{y}}_{k, l}\right),
\end{aligned}
$$

where $\theta_{k, l}$ is the measurement-to-extended target assignment, i.e., its value gives the extended target from which the measurement stems (see also [18], [19]). The first expression $f_{k, l-1}\left(\underline{x}_{k}^{A} \mid \theta_{k, l}, \underline{\hat{y}}_{k, l}\right)$ in (8) is the posterior density for given measurement-to-target association, which can be directly computed according to the measurement equation (6) derived for single extended targets (see also [4]). ${ }^{1}$

The second expression $f_{k, l-1}\left(\theta_{k, l} \mid \underline{y}_{k, l}\right)$ in (8) is the posterior association probability, which can be reformulated according to Bayes' Theorem to

$$
f_{k, l-1}\left(\theta_{k, l} \mid \underline{\hat{y}}_{k, l}\right)=c \cdot f_{k, l-1}\left(\underline{\hat{y}}_{k, l} \mid \theta_{k, l}\right) \cdot f\left(\theta_{k, l}\right),
$$

where $f\left(\theta_{k, l}\right)$ is the association prior of the Mixture RHM as given by Definition IV.1 and the predicted measurement $f_{k, l-1}\left(\underline{\hat{y}}_{k, l} \mid \theta_{k, l}\right)$ can be computed based on the measurement equation (6).

Implementation as Gaussian Filter: The above presented formal Bayesian filter can be implemented as Gaussian-assumed filter, i.e., all probability densities are approximated with a Gaussian density, i.e., $f_{k, l}\left(\underline{x}_{k}\right) \approx$ $\mathcal{N}\left(\underline{x}_{k}-\underline{\mu}_{k, l}^{x}, \Sigma_{k, l}^{x}\right)$. Then, the posterior density for given measurement-to-target association $f_{k, l-1}\left(\underline{x}_{k}^{A} \mid \theta_{k, l}, \underline{\hat{y}}_{k, l}\right)$ in (8) can be computed by using the measurement equation (6) and a Gaussian filter such as the Unscented Kalman filter [20] as described in [4]. The posterior density (8) is a Gaussian mixture with components $f_{k, l-1}\left(\underline{x}_{k}^{A} \mid \theta_{k, l}, \underline{y}_{k, l}\right)$ and weights $f_{k, l-1}\left(\theta_{k, l} \mid \underline{\hat{y}}_{k, l}\right)$. If we are aiming at a Gaussian filter, it can be approximated with a single Gaussian density by means of moment matching in the style of the Joint Probabilistic Data Association Filter (JPDAF) [18]. In general, more complex techniques such as a Multi-Hypothesis-Tracker (MHT) [21], which manages all feasible hypothesis over time, could also be used.

\section{EVALUATION}

In the following, we provide an evaluation of the introduced method for tracking multiple extended objects based on Mixture RHMs.

\section{A. Two Static Extended Objects}

First, two extended targets with a fixed position and shape are considered (see Fig. 5a). Measurements are generated sequentially from the targets, where each target is the source of the measurement with equal probability. The measurement sources are drawn uniformly from the target surfaces. The measurement noise of a particular measurement is $\Sigma_{k, 1}^{v}=\operatorname{diag}(0.8,0.8)$ with probability 0.4 and $\Sigma_{k, 1}^{v}=\operatorname{diag}(0.3,0.3)$ with probability 0.6 . Based on the sequentially arriving noisy position measurements, the shapes of the two targets are estimated with a Mixture RHM for star-convex shapes implemented according to the Gaussian-assumed Bayesian estimation procedure described in this paper. The radius function is represented with 11 Fourier descriptors and the squared scaling factor is Gaussian distributed with mean 0.7 and variance 0.03 for both

\footnotetext{
${ }^{1}$ Note that this is a slight abuse of notation, as according to (6) we in fact condition on the pseudo-measurement 0 and not on the measurement itself.
} 
targets. The shape parameters are a priori set to a Gaussian with mean $[0.5,2.5,2,0, \ldots, 0]^{T}$ for the first target and $[-0.5,-3.5,3,0, \ldots, 0]^{T}$ and $[0.5,0.5,3,0, \ldots, 0]^{T}$ for the second target. The covariance matrices are for both shape parameters $\operatorname{diag}(0.05,0.05,0.01,0.01, \ldots, 0.01)$. These are uncertain circles with radius 1.5 located at the positions $[0.5,0.5]^{T}$ and $[5.5,2.5]^{T}$.

The shape estimates are averaged over 20 Monte Carlo runs. The average shape estimates for the targets are depicted in Fig. 5c after having received 300 measurements. Note that the uncertainty of the shape estimates has not been plotted. For illustrating the magnitude of the measurement noise, the measurements of an example run are depicted in Fig. 5b. Note that the estimator processes measurements recursively, because in practical applications the target state evolves over time. It can be seen that for both targets, the shape is estimated precisely, although it is not known from which target a particular measurement stems. This example shows that detailed object information can be extracted with Mixture RHMs for multiple star-convex shapes, when the origin of the measurement is unknown.

\section{B. Tracking Two Moving Extended Objects}

In the second scenario, two extended objects are tracked based on a Mixture RHM and a constant velocity model for the target motion. The extended objects move along the trajectory depicted in Fig. 6, i.e., they start well-separated near the origin, then approach each other, and perform a turn. At each time step, the total number of measurements received from the targets is Poisson distributed with mean 20 , where each target is the source of a measurement with the same probability. The measurement noise is zero-mean Gaussian with covariance matrix $\Sigma_{k, l}^{v}=\operatorname{diag}(0.2,0.2)$ with probability 0.5 and $\Sigma_{k, l}^{v}=\operatorname{diag}(0.8,0.8)$ with probability 0.5 . The state vector of a single target $i \in\{1,2\}$ is given by $\underline{\boldsymbol{x}}_{k}^{i}=\left[\left(\underline{\boldsymbol{p}}_{k}^{i}\right)^{T}, \dot{\boldsymbol{x}}_{k}^{i}, \dot{\boldsymbol{y}}_{k}^{i}\right]^{T}$, where $\left[\dot{\boldsymbol{x}}_{k}^{i}, \dot{\boldsymbol{y}}_{k}^{i}\right]^{T}$ is the velocity vector and $\underline{\boldsymbol{p}}_{k}^{i}$ are the shape parameters given by 11 Fourier descriptors. As the extended object is assumed to evolve according to a constant velocity model, the system equation is $\underline{\boldsymbol{x}}_{k+1}^{i}=\mathbf{A}_{k} \underline{\boldsymbol{x}}_{k}^{i}+\underline{\boldsymbol{w}}_{k}^{i}$. The system matrix is $\mathbf{A}_{k}=\operatorname{diag}\left(\mathbf{I}_{11}, \mathbf{A}_{k}^{c v}\right)$ with $\mathbf{A}_{k}^{c v}=\left[\begin{array}{cc}\mathbf{I}_{2} & T \mathbf{I}_{2} \\ \mathbf{0}_{2} & \mathbf{I}_{2}\end{array}\right]$, where $\mathbf{I}_{11}$ is an identity matrix of dimension $11, \mathbf{I}_{2}$ is a twodimensional identity matrix, and $\mathbf{0}_{2}$ is a two-dimensional zero matrix. The system noise $\underline{\boldsymbol{w}}_{k}^{i}$ is zero-mean Gaussian noise with covariance matrix $\mathbf{C}_{k}^{w}=\operatorname{diag}\left(0.03 \cdot \mathbf{I}_{11}, \mathbf{C}_{k}^{c v}\right)$ with $\mathbf{C}_{k}^{c v}=0.005\left[\begin{array}{cc}\frac{T^{3}}{3} \mathbf{I}_{2} & \frac{T^{2}}{2} \mathbf{I}_{2} \\ \frac{T^{2}}{2} \mathbf{I}_{2} & T \mathbf{I}_{2}\end{array}\right]$. Hence, the center of the object evolves according to a constant velocity model and the shape parameters become more uncertain over time in order to capture shape changes. A Gaussian scaling factor with mean 0.7 and variance 0.03 is used. The target shapes are tracked with a Mixture $R H M$ implemented by using a UKF [20].

The estimated shapes are depicted in Fig. 7 for two snippets of the trajectory (averaged over 20 runs). It can

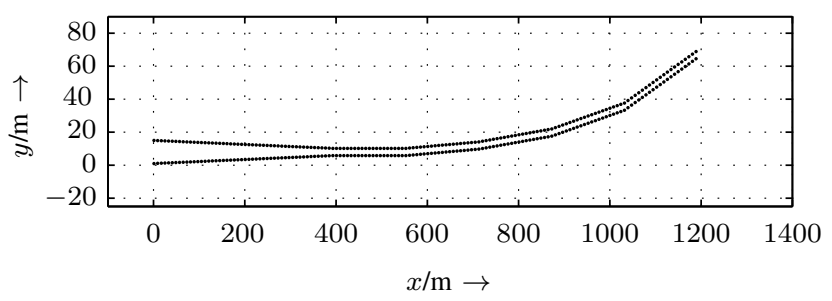

Fig. 6: Trajectory of the two extended targets.

be seen that the shapes of the extended objects are tracked well, even when the targets move very close to each other and change the orientation. Altogether, this example demonstrates that Mixture RHMs are feasible for tracking starconvex shape approximations of multiple extended objects that move according to a constant velocity model.

\section{CONCLUSiOnS AND Future WORK}

We have presented a new concept called Mixture Random Hypersurface Models for modeling spatially distributed noise-corrupted data. In this work, we have used the concept for tracking multiple extended objects based on noisy position measurements. For this purpose, we have derived a Gaussian state estimators for estimating the shape of multiple extended objects. Simulations demonstrate the feasibility of the approach by means of a typical tracking scenario.

Prospective work will focus on making the presented tracking algorithm robust to clutter measurements that do not stem from a target. Furthermore, we will incorporate statistical knowledge about the number of measurements generated by a target. Mixture RHMs can also be used to compose a single extended objects of several connected Random Hypersurface Models, which will provide even more detailed shape information. Finally, we believe that Mixture RHMs represent a fundamental concept for modeling spatial data, which can also be used for other applications such as cluster analysis.

\section{REFERENCES}

[1] K. Gilholm and D. Salmond, "Spatial Distribution Model for Tracking Extended Objects," IEE Proceedings on Radar, Sonar and Navigation, vol. 152, no. 5, pp. 364-371, October 2005.

[2] W. Koch and R. Saul, "A Bayesian Approach to Extended Object Tracking and Tracking of Loosely Structured Target Groups," 8th International Conference on Information Fusion, vol. 1, July 2005.

[3] M. Baum, B. Noack, and U. D. Hanebeck, "Extended Object and Group Tracking with Elliptic Random Hypersurface Models," in Proceedings of the 13th International Conference on Information Fusion (Fusion 2010), Edinburgh, United Kingdom, Jul. 2010.

[4] M. Baum and U. D. Hanebeck, "Shape Tracking of Extended Objects and Group Targets with Star-Convex RHMs," in Proceedings of the 14th International Conference on Information Fusion (Fusion 2011), Chicago, Illinois, USA, Jul. 2011.

[5] K. Gilholm, S. Godsill, S. Maskell, and D. Salmond, "Poisson Models for Extended Target and Group Tracking," in SPIE: Signal and Data Processing of Small Targets, 2005.

[6] Y. Boers, H. Driessen, J. Torstensson, M. Trieb, R. Karlsson, and F. Gustafsson, "Track-Before-Detect Algorithm for Tracking Extended Targets," IEE Proceedings on Radar, Sonar and Navigation, vol. 153, no. 4, pp. 345 -351, August 2006.

[7] R. Mahler, "PHD Filters for Nonstandard Targets, I: Extended Targets," in Proceedings of the 12th International Conference on Information Fusion (Fusion 2009), Seattle, Washington, July 2009. 


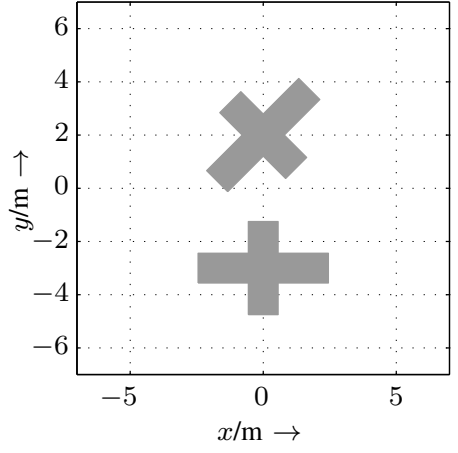

(a) Two extended targets.

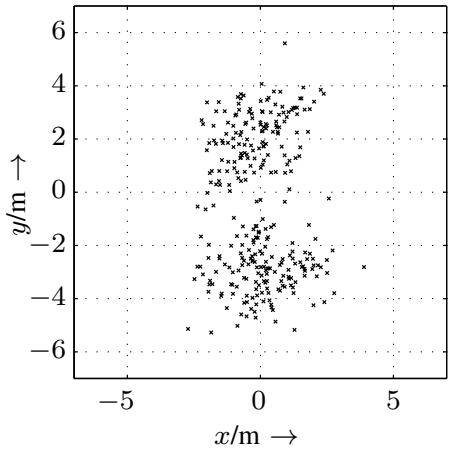

(b) Measurements of an example run.

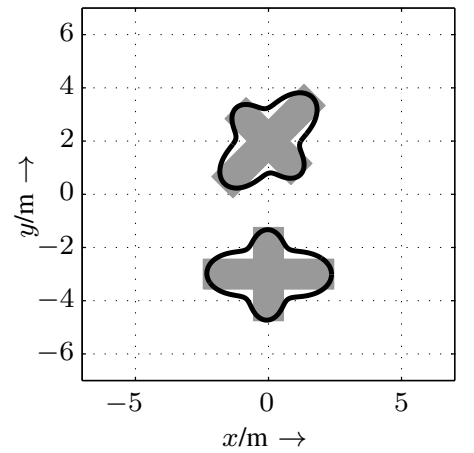

(c) Estimated shapes averaged over 20 runs.

Fig. 5: Non-moving targets: Simulation results.

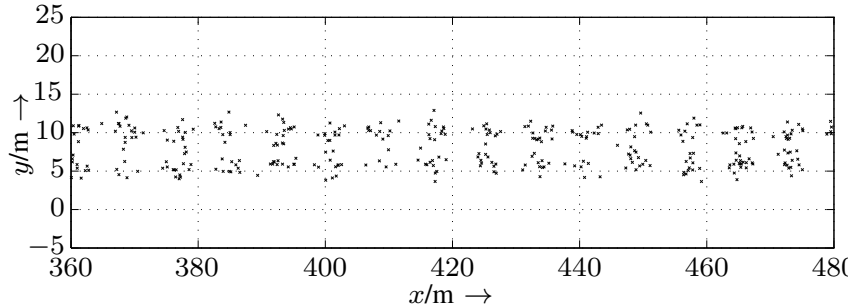

(a) Measurements of an example run (Snippet 1).

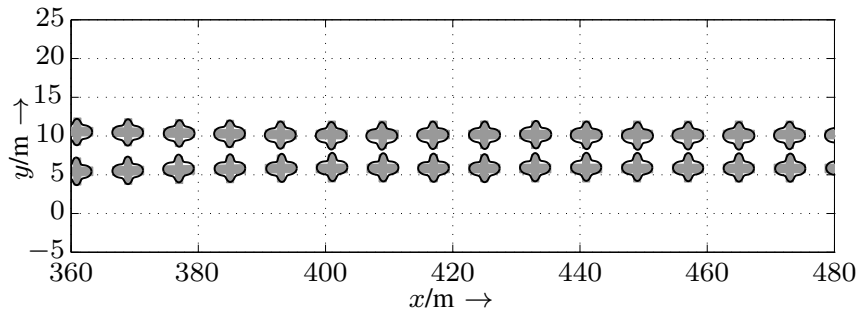

(c) Average shape estimates and true extended target (Snippet 1).

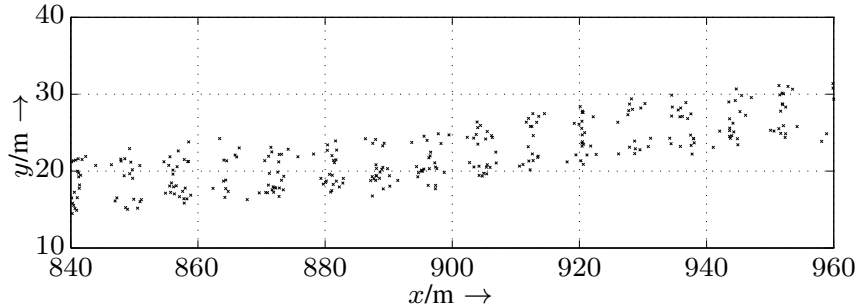

(b) Measurements of an example run (Snippet 2).

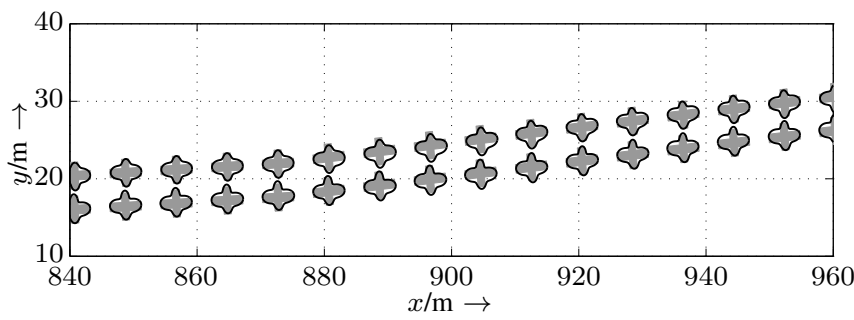

(d) Average shape estimates and true extended target (Snippet 2).

Fig. 7: Tracking two extended objects with a Mixture RHM: Simulation results. Several time steps are plotted in one figure.

[8] K. Granström, C. Lundquist, and U. Orguner, "A Gaussian Mixture PHD filter for Extended Target Tracking," in Proceedings of the 13th International Conference on Information Fusion (Fusion 2010), Edinburgh, Scotland, Jul. 2010.

[9] N. Petrov, L. Mihaylova, A. Gning, and D. Angelova, "A Novel Sequential Monte Carlo Approach for Extended Object Tracking Based on Border Parameterisation," in Proceedings of the 14th International Conference on Information Fusion (Fusion 2011), Chicago, Illinois, USA, Jul. 2011.

[10] J. W. Koch, "Bayesian Approach to Extended Object and Cluster Tracking using Random Matrices," IEEE Transactions on Aerospace and Electronic Systems, vol. 44, no. 3, pp. 1042-1059, July 2008.

[11] M. Feldmann, D. Franken, and W. Koch, "Tracking of Extended Objects and Group Targets using Random Matrices," IEEE Transactions on Signal Processing, vol. PP, no. 99, p. 1, 2010.

[12] M. Wieneke and W. Koch, "Probabilistic Tracking of Multiple Extended Targets using Random Matrices," in SPIE: Signal and Data Processing of Small Targets, vol. 7698, 2010.

[13] M. Baum, M. Feldmann, D. Fränken, U. D. Hanebeck, and W. Koch, "Extended Object and Group Tracking: A Comparison of Random Matrices and Random Hypersurface Models," in Proceedings of the IEEE ISIF Workshop on Sensor Data Fusion: Trends, Solutions, Applications (SDF 2010), Leipzig, Germany, Oct. 2010.

[14] M. Baum and U. D. Hanebeck, "Random Hypersurface Models for Extended Object Tracking," in Proceedings of the 9th IEEE Interna- tional Symposium on Signal Processing and Information Technology (ISSPIT 2009), Ajman, United Arab Emirates, Dec. 2009.

[15] D. Zhang and G. Lu, "Study and Evaluation of Different Fourier Methods for Image Retrieval," Image and Vision Computing, vol. 23, no. 1 , pp. $33-49,2005$.

[16] R. Jain, R. Kasturi, and B. G. Schunck, Machine Vision. New York, NY, USA: McGraw-Hill, Inc., 1995.

[17] D. Crouse, M. Guerriero, P. Willett, R. Streit, and D. Dunham, "A Look at the PMHT," in Proceedings of the 12th International Conference on Information Fusion (Fusion 2009), July 2009.

[18] Y. Bar-Shalom, F. Daum, and J. Huang, "The Probabilistic Data Association Filter," IEEE Control Systems Magazine, vol. 29, no. 6 , pp. $82-100$, Decembre 2009.

[19] J. Vermaak, S. Godsill, and P. Perez, "Monte Carlo Filtering for Multi Target Tracking and Data Association," IEEE Transactions on Aerospace and Electronic Systems, vol. 41, no. 1, pp. $309-332$, January 2005

[20] D. Simon, Optimal State Estimation: Kalman, H Infinity, and Nonlinear Approaches, 1st ed. Wiley \& Sons, August 2006.

[21] S. Blackman, "Multiple Hypothesis Tracking for Multiple Target Tracking," IEEE Aerospace and Electronic Systems Magazine, vol. 19, no. 1, pp. $5-18$, January 2004. 